\title{
THE GLEASON-KAHANE-ŻELAZKO THEOREM AND ITS GENERALIZATIONS
}

\author{
A. R. SOUROUR \\ Department of Mathematics and Statistics, University of Victoria \\ Victoria, B.C., V8W 3P4, Canada
}

\begin{abstract}
This expository article deals with results surrounding the following question Which pairs of Banach algebras $A$ and $B$ have the property that every unital invertibility preserving linear map from $A$ to $B$ is a Jordan homomorphism?

In this article, all Banach algebras are over the field of complex numbers $\mathbb{C}$ and are assumed to have an identity 1 . The spectrum of an element $a$ is denoted by $\sigma(a)$. A map $\phi$ from an algebra $A$ to an algebra $B$ is called unital if $\phi(1)=1$, is called invertibility preserving if $\phi(a)$ is invertible in $B$ for every invertible element $a \in A$, and is called a Jordan homomorphism if $\phi(a b+b a)=\phi(a) \phi(b)$ for all $a$ and $b \in A$, or equivalently $\phi\left(a^{2}\right)=(\phi(a))^{2}$ for every $a \in A$. It is obvious that every homomorphism and every anti-homomorphism is a Jordan homomorphism. (A linear map $\phi$ is called an anti-homomorphism if $\phi(a b)=\phi(b) \phi(a)$.)

There is a history of research activity centered around the question of when invertibility preserving linear maps are Jordan homomorphisms. One of the earliest results in this area is the following, which was obtained independently by Gleason [5] and Kahane and Żelazko [9].
\end{abstract}

THEOREM 1 ([5], [9]). Let $\phi$ be a unital invertibility preserving linear map from a commutative Banach algebra $A$ to a commutative semisimple Banach algebra $B$. Then $\phi$ is multiplicative.

R e m a r ks. 1. Żelazko [15] has shown that the conclusion of the above theorem also holds for noncommutative $A$.

2. It is shown in [5] and [9] that the proof of the above theorem reduces easily

1991 Mathematics Subject Classification: Primary 47B48; Secondary 46H99.

The paper is in final form and no version of it will be published elsewhere.

Research supported by grants from NSERC and from the University of Victoria. 
to the case where $B=\mathbb{C}$. In this case the condition on $\phi$ is equivalent to the condition that $\sigma(\phi(a)) \subset \sigma(a)$ for every $a \in A$, and the result can be stated as a characterization of maximal ideals.

The next theorem, dealing with matrix algebras, is due to Marcus and Purves [11], and in the case of bijective mappings to Dieudonné [3], who considers "semilinear" maps of matrix algebras over arbitrary fields which preserve noninvertibility. In what follows, we denote the transpose of a matrix $x$ by $x^{t}$.

THEOREM 2 ([3], [11]). Let $\phi$ be a unital invertibility preserving linear map on $M_{n}$, the algebra of all $n \times n$ complex matrices. Then $\phi$ takes one of the following forms:

$$
\phi(x)=a x a^{-1} \quad \text { or } \quad \phi(x)=a x^{t} a^{-1},
$$

for some invertible element $a \in M_{n}$.

Re mark. The conclusion of Theorem 2 is equivalent to asserting that $\phi$ is a Jordan isomorphism. This follows from a result of Herstein [6, pp. 47-51] which states that every Jordan homomorphism of rings whose range is a prime ring is either a homomorphism or an anti-homomorphism, together with the classical result that every automorphism of $M_{n}$ is inner. (Recall that a ring $R$ is called prime if $a R b=0$ implies that $a=0$ or $b=0$.)

Motivated by the results above, Kaplansky [10] raised the question of when must additive invertibility preserving maps on rings be homomorphisms (or Jordan homomorphism) $\left(^{1}\right)$. Of course this is not always the case for arbitrary rings (see [10]). There are also counterexamples for linear unital maps on Banach algebras. Indeed, if $A$ is obtained from a radical algebra by adjoining an identity, then any unital linear map on $A$ that maps the radical part to itself preserves invertibility but is rarely multiplicative. We give below a concrete example. Another example is given by Aupetit in [1, p. 28].

EXAMPLE. Let $A$ be the algebra of upper triangular $n \times n$ matrices with $n \geq 3$, and let $\phi\left(a_{i j}\right)=\left(b_{i j}\right)$ where $b_{12}=a_{13}, b_{13}=a_{12}$, and $b_{i j}=a_{i j}$ otherwise. It is immediate that $\phi$ is unital and preserves invertibility. However, if $c$ is the matrix given by $c_{23}=c_{13}=1, c_{i j}=0$ otherwise, then $\phi\left(c^{2}\right)=0$ but $(\phi(c))^{2} \neq 0$, and so $\phi$ is not a Jordan homomorphism. Observe that, as in Aupetit's example, $(\phi(c))^{2}-\phi\left(c^{2}\right)$ is nilpotent.

The following theorem of Aupetit [1] is a generalization of Theorems 1 and 2 .

Theorem 3 ([1]). Let $A$ and $B$ be Banach algebras and assume that $B$ has a separating family of irreducible finite-dimensional representations. If $\phi: A \rightarrow B$ is a surjective, unital, invertibility preserving linear map, then $\phi$ is a Jordan homomorphism.

$\left({ }^{1}\right)$ Editorial note: See also footnote $\left({ }^{2}\right)$ in the paper of M. Mathieu in this volume. 
The next two results deal with *-algebras and *-linear maps. The von Neumann algebra result is due to Russo [13] and the $C^{*}$-algebra result is due to Choi-Hadwin-Nordgren-Radjavi-Rosenthal [2]. We state them together.

Theorem 4 ([2], [13]). Let $A$ and $B$ be $C^{*}$-algebras and let $\phi: A \rightarrow B$ be a unital invertibility preserving linear map such that $\phi\left(a^{*}\right)=(\phi(a))^{*}$, for every $a \in A$. Then $\phi$ is a Jordan homomorphism provided that either

(i) $A$ and $B$ are von Neumann algebras, or

(ii) $\phi$ is surjective and continuous.

Remark. A counterexample in [2] shows that the result above is false when the range of $\phi$ is not a $C^{*}$-algebra. In that example, $\phi$ has a dense range.

The next result generalizes Dieudonné's to infinite-dimensional spaces. The algebra of all bounded operators on a Banach space $X$ is denoted by $\mathcal{B}(X)$.

TheOREM 5 ([14]). Let $X$ and $Y$ be Banach spaces over the complex field and let $\phi$ be a unital bijective linear map from $\mathcal{B}(X)$ to $\mathcal{B}(Y)$. The following conditions are equivalent:

(a) $\phi$ preserves invertibility.

(b) $\phi$ is a Jordan isomorphism.

(c) $\phi$ is either an isomorphism or an anti-isomorphism.

(d) Either

(i) $Y$ is isomorphic to $X$ and $\phi(T)=A^{-1} T A$ for every $T \in \mathcal{B}(X)$ where $A$ is an isomorphism from $Y$ to $X$, or

(ii) $Y$ is isomorphic to $X^{*}$ and $\phi(T)=B^{-1} T^{*} B$ for every $T \in \mathcal{B}(X)$ where $B$ is an isomorphism from $Y$ to $X^{*}$.

The proof of Theorem 5 uses the following characterizations of rank one operators, which are also of independent interest.

Proposition 1. Let $R \in \mathcal{B}(X), R \neq 0$. The following are equivalent.

(i) $R$ has rank one.

(ii) $\sigma(T+\alpha R) \cap \sigma(T+\beta R) \subseteq \sigma(T)$, for every $T \in \mathcal{B}(X)$, and every scalars $\alpha$ and $\beta$, where $\alpha \neq \beta$.

(iii) For every $T \in \mathcal{B}(X)$, there exists a compact subset $K_{T}$ of the complex plane such that

$$
\sigma(T+\alpha R) \cap \sigma(T+\beta R) \subseteq K_{T},
$$

for every $T \in \mathcal{B}(X)$, and every scalars $\alpha \neq \beta$.

Remarks. 1. The equivalence of (i) and (ii) is given in [8] and is used there to characterize spectrum preserving linear maps. The stronger equivalence of (i) and (iii) is what is needed to prove Theorem 5 .

2. Function theory seems to play a role in several proofs. The proof of Proposition 1 in [14], which is too technical to include here, uses Picard's Theorem, 
and the proof of the main result in [9] uses Hadamard's Theorem. However, an elementary proof of Theorem 1 can be found in [12].

Outline of proof of Theorem 5 . What follows is a brief outline with most details omitted. First we introduce some notation. The duality between a Banach space and its dual is denoted by $\langle$,$\rangle . The rank one operator u \rightarrow f(u) x$ is denoted by $x \otimes f$.

The implications $(d) \Rightarrow(c) \Rightarrow(b) \Rightarrow(a)$ are easy. To show that $(\mathrm{a}) \Rightarrow(d)$, assume that condition (a) is satisfied. It follows that

$$
\sigma(\phi(T)) \subseteq \sigma(T),
$$

for every $T \in \mathcal{B}(X)$. Condition (iii) of Proposition 1 implies that $\phi$ sends rank one operators to rank one operators. Thus if $x \in X$ and $f \in X^{*}$, then $\phi(x \otimes f)=y \otimes g$ for some $y \in Y$ and $g \in Y^{*}$. It is not too difficult to prove that $y$ depends linearly only on one of $x$ and $f$, and that $g$ depends only on the other variable. Therefore

$$
\phi(x \otimes f)=A x \otimes C f,
$$

or

$$
\phi(x \otimes f)=B x \otimes D f,
$$

where $A: X \rightarrow Y$ and $C: X^{*} \rightarrow Y^{*}$, or $B: X^{*} \rightarrow Y$ and $D: X \rightarrow Y^{*}$, are linear transformations.

Next we use the fact (see [8]) that if $\lambda \notin \sigma(T)$, then $\lambda \in \sigma(T+x \otimes f)$ if and only if $\left\langle(\lambda-T)^{-1} x, f\right\rangle=1$. This, together with the spectral inclusion $(\dagger)$, implies that

$$
\left\langle(1-z T)^{-1} x, f\right\rangle=\left\langle(1-z \phi(T))^{-1} C x, D f\right\rangle,
$$

for every $z$ in a deleted neighbourhood of 0 . These equations and the closed graph theorem show that $A$ and $C$ (or $B$ and $D$ ) are bounded. Taking the limit as $z \rightarrow 0$, we see that $C=\left(A^{-1}\right)^{*}$ or that $D=\left(B^{-1}\right)^{*} \mid X$. Taking the derivative at 0 , we obtain the forms of condition (d) in Theorem 5 .

Remarks. 1. The map $\phi$ in Theorem 5 is not assumed to be continuous in any topology. Of course, the conclusion of the theorem implies that if it preserves invertibility, then it must be continuous when $\mathcal{B}(X)$ and $\mathcal{B}(Y)$ are both equipped with any of the standard operator topologies.

2. Weakly continuous rank preserving maps on $\mathcal{B}(X)$ are characterized in [7].

3. The equivalence of conditions (c) and (d) in Theorem 5 implies that every isomorphism of $\mathcal{B}(X)$ is inner, a result due to Eidelheit [4].

4. The equivalence of conditions (b) and (c) in Theorem 5 also follows from Herstein's Theorem [6, pp. 47-51], as indicated in the remark following Theorem 2 above.

We note that Proposition 1 can be generalized, as stated below, to a characterization of operators having rank less than $n$ for a fixed positive integer $n$. 
Proposition 2. The operator $R$ has rank less than $n$ if and only if for every $T \in \mathcal{B}(X)$, there exists a compact subset $K_{T}$ of the complex plane such that

$$
\bigcap_{1 \leq j \leq n} \sigma\left(T+\alpha_{j} R\right) \subseteq K_{T}
$$

for every $T \in \mathcal{B}(X)$, and any $n$ distinct scalars $\alpha_{j}, j=1, \ldots, n$.

We end by asking a more concrete question than the one in the Abstract. Let $A$ be semisimple Banach algebra and $\phi$ a bijective linear map on $A$ that preserves invertibility; must $\phi$ be a Jordan isomorphism?

Acknowledgements. The author is grateful to Professor J. Zemánek for bringing to his attention references [7] and [12].

\section{References}

[1] B. Aupetit, Propriétés Spectrales des Algèbres des Banach, Lecture Notes in Math. 735, Springer, 1979.

[2] M.-D. Choi, D. Hadwin, E. Nordgren, H. Radjavi and P. Rosenthal, On positive linear maps preserving invertibility, J. Funct. Anal. 59 (1984), 462-469.

[3] J. Dieudonné, Sur une généralisation du groupe orthogonal à quatre variables, Arch. Math. (Basel) 1 (1949), 282-287.

[4] M. Eidelheit, On isomorphisms of rings of linear operators, Studia Math. 9 (1940), $97-105$.

[5] A. Gleason, A characterization of maximal ideals, J. Analyse Math. 19 (1967), 171-172

[6] I. N. Herstein, Topics in Ring Theory, University of Chicago Press, Chicago, 1969.

[7] J.-C. Hou, Rank preserving linear maps on $\mathcal{B}(X)$, Science in China (Series A) 32 (1989), 929-940.

[8] A. Jafarian and A. R. Sourour, Spectrum preserving linear maps, J. Funct. Anal. 66 (1986), 255-261.

[9] J.-P. Kahane and W. Żelazko, A characterization of maximal ideals in commutative Banach algebras, Studia Math. 29 (1968), 339-343.

[10] I. Kaplansky, Algebraic and Analytic Aspects of Operator Algebras, Amer. Math. Soc., Providence, 1970.

[11] M. Marcus and R. Purves, Linear transformations on algebras of matrices: The invariance of the elementary symmetric functions, Canad. J. Math. 11 (1959), 383-396.

[12] M. Roitman and Y. Sternfeld, When is a linear functional multiplicative?, Trans. Amer. Math. Soc. 267 (1981), 111-124.

[13] B. Russo, Linear mappings of operator algebras, Proc. Amer. Math. Soc. 17 (1966), $1019-1022$.

[14] A. R. Sourour, Invertibility preserving linear maps, preprint, 1992.

[15] W. Żelazko, A characterization of multiplicative linear functionals in complex Banach algebras, Studia Math. 30 (1968), 83-85. 